\title{
Impacto de Tres Modelos de Enseñanza de la Asignatura Botánica General sobre el Rendimiento Académico de los Estudiantes
}

\author{
Aixa O. Rivero-Guerra \\ Universidad Estatal Amazónica, Departamento de Ciencias de la Vida, Programa de Ecosistemas, Biodiversidad y \\ Conservación de Especies, Paso Lateral Puyo-Tena, Km 2 1/2, Puyo, Pastaza, Ecuador. \\ (e-mail: rivero-guerra@hotmail.com; arivero@uea.edu.ec)
}

Recibido Jul. 31, 2018; Aceptado Oct. 4, 2018; Versión final Ene. 11, 2019, Publicado Jun. 2019

\begin{abstract}
Resumen
Se evalúan tres modelos para la enseñanza de la asignatura de Botánica General, que se imparte durante el primer semestre en la carrera de Ingeniería Agropecuaria en la Universidad Estatal Amazónica (Ecuador). Se determinan los estilos y estrategias de aprendizaje de los 29 estudiantes matriculados, además del efecto del modelo pedagógico en sus calificaciones. Las tres unidades de la asignatura se imparten con la siguiente metodología: i) La Célula (modelo tradicional), ii) Tejidos Vegetales (modelo mixto), y iii) Organografía y Sistemas de Dispersión del Polen, Frutos y Semillas (modelo de aula invertida). El estilo de aprendizaje preferente es el reflexivo, y existe un equilibrio en el número de estudiantes pragmáticos, teóricos y prácticos. La metodología de aula invertida presenta un impacto significativo en el rendimiento académico, aunque el nivel de satisfacción de los estudiantes es estable durante todo el semestre. Sus estrategias de aprendizaje cooperativo, profundo y autónomo están en fase inicial de desarrollo.
\end{abstract}

Palabras clave: estilos de aprendizaje; estrategias de aprendizaje; evaluación formativa; evaluación sumativa; aula invertida

\section{Impact of Three Teaching Models in University General Botany Courses on the Academic Results of Students}

\begin{abstract}
Three models for the teaching of the General Botany course delivered during the first semester of the Agricultural Engineering career at the Amazonian State University (Ecuador) are evaluated. The learning styles and strategies of the 29 enrolled students are determined, as well as the effect of the pedagogical model on their grades. Three Units have been developed for this teaching plan using different methodologies: i) The Cell (traditional model), ii) Vegetable Tissues (mixed model), and iii) Organography and Systems for the Dispersion of Pollen, Fruits and Seeds (the flipped classroom model). The preferred teaching model is the reflexive one, which gives a good balance between pragmatic, theoretical and practical students. The methodology of flipped classroom yields a significant impact in the development of academic results, which satisfied the students during the whole semester. The cooperative, in-depth, and autonomous learning strategies are in the first phase of development.
\end{abstract}

Keywords: learning styles; learning strategies; formative evaluation; summative evaluation; flipped classroom 


\section{INTRODUCCIÓN}

La Botánica, como ciencia, tuvo un marcado auge del siglo XVII al XIX (Rivero-Guerra, 2013). La enseñanza teórica y práctica de la Botánica mermó en las últimas décadas, al ser desplazada del currículo de las carreras universitarias (Byng et al., 2018), y los estudiantes de Biología y de especialidades afines muestran limitados conocimientos en esta materia. En este contexto, existen varias razones que justifican este decreciente interés por parte de los estudiantes y las instituciones por esta ciencia. Desde la educación primaria se detecta una desconexión de los niños con la naturaleza y la biodiversidad y las escuelas no propician los entornos asequibles que garanticen la conectividad con diferencias significativas entre la educación pública y privada (Bermúdez et al., 2018). En Botánica, se precisa alcanzar un alto nivel de competencia que involucre el conocimiento de las plantas en su entorno, relacionándola con el ecosistema donde habitan. En un curso de Botánica el estudiante se apropia de habilidades para observar, describir, analizar, diferenciar y clasificar algas, plantas y hongos, además de conocer que la evolución de los caracteres morfológicos, fisiológicos, reproductivos y genéticos no ocurre de forma aislada en la naturaleza, sino en interacción con otros organismos vivos y con el ambiente; así como sus propiedades fitoquímicas, sin olvidar la interrelación plantas-hombre-sociedad. Además de vincular la asignatura con otras ciencias afines y con el futuro perfil profesional de los egresados. Diversos autores proponen la enseñanza de diferentes conceptos que se manejan en el estudio de la biodiversidad de plantas desde un enfoque holístico, interdisciplinar, científico, ecológico, integrador, sistémico, evolutivo, ético y socio-económico (García-Vázquez y Méndez-Pupo, 2017). El profesor de Botánica debe de promover la puesta en marcha de estrategias didácticas fuera del aula para suscitar un cambio en el comportamiento del estudiante hacia la naturaleza e incentivar significativamente su aprendizaje, al impulsar su interés por resolver problemas necesarios como la conservación de las especies y el medio en condiciones naturales, además de asumir una actitud responsable en relación a la contaminación ambiental y el uso sostenible de los recursos de la naturaleza (Montilla, 2008). Lo que propicia la puesta en práctica de la creatividad y el pensamiento abstracto para la construcción de conocimiento.

Se observan aún inconsistencias teóricas y metodológicas en la práctica y los estudiantes demandan prácticas de campo o de laboratorio inmediatamente después de la lección teórica (Santos da Silva et al., 2016; García-Vázquez y Méndez-Pupo, 2017). Ambas acercan al estudiante al conocimiento científico, al aprender y aplicar activamente los contenidos procedimentales cognitivos y metacognitivos (Lazzari et al., 2017), además del desarrollo de habilidades en correspondencia con el modelo constructivista al ser protagonista de su proceso de enseñanza-aprendizaje, que favorece el desarrollo del pensamiento hipotético deductivo y lo guía hacia un posible futuro profesional (Yan, 2014; Pérez et al., 2008). Las prácticas tienen un enfoque investigador y multidisciplinar al articular experiencias, experimentos, observación, técnicas manipulativas, ejercicios prácticos, elaboración y validación de hipótesis, análisis, interpretación y elaboración de resultados, que posibilitan estudiar los fenómenos biológicos a nivel microscópico y macroscópico. Todos estos procesos estimulan la interacción, la participación, y la discusión o debate así como la generación de distintos cuerpos de conocimiento significativos (Montilla, 2008). Los datos obtenidos en el laboratorio y/o en el campo conllevan a la elaboración de modelos teóricos o a perfeccionar los existentes; a más de ser pertinentes para clarificar y corregir los conceptos o percepciones erróneas.

Dada la complejidad de los contenidos que se imparten en Botánica, la aplicación del modelo pedagógico de clase invertida otorga nuevas posibilidades de aprendizaje significativo (Rivero-Guerra, 2018), en la que el profesor planifica, desarrolla y evalúa de forma estratégica los contenidos de la asignatura (Uriarte, 1996). En este modelo el rol profesor-estudiante se invierte y los estudiantes deben de trabajar de forma autónoma en tareas o actividades previamente asignadas por el docente (Jensen, 2018), que tienen un fuerte soporte electrónico y serán objeto de estudio y profundización en la clase presencial (Sabater-Mateu et al., 2017). Las actividades extraclase que se designan son de un mayor nivel cognitivo como analizar, evaluar y crear conocimiento (Hacisalihoglu et al., 2018). Durante la clase presencial se debate, discuten o ejercitan los contenidos a tratar, con una participación activa de todos los estudiantes al avivar su pensamiento crítico y complejo e intensificar el trabajo en equipo (Williams et al., 2018). Las tutorías presenciales y personalizadas contribuyen significativamente a guiar al estudiante hacia el cumplimiento de los objetivos, al proporcionar las herramientas necesarias para que las adapte a su estilo de aprendizaje, y con ello alcanzar las metas establecidas por la asignatura y las suyas propias, incluso superarlas (Arbizu y Lobato, 2003; Chalmers et al., 2017). De este modo la educación y la enseñanza son procesos más humanísticos, se trata de que el estudiante aprenda más y mejor (Hacisalihoglu et al., 2018). 
Este método contrasta con la docencia tradicional, que en ocasiones resulta excesivamente memorística, principalmente a través de la reproducción de los contenidos expuestos en un libro didáctico, aunque esto no implica que no se lleven a cabo trabajos prácticos, seminarios y debates (Limniou et al., 2018). A través de la clase invertida se fomenta el aprendizaje proactivo en dos direcciones, dentro y fuera del aula, la motivación de los estudiantes y el incremento de la asistencia a clase. Esto favorece el desarrollo de competencias transversales y da paso a que el docente pueda monitorear en tiempo real los errores o incongruencias en el proceso de aprendizaje. Las implicaciones son relevantes, permanente retroalimentación por parte del docente, evaluación asidua y mejora continua en el tiempo a favor de la educación universitaria (Rivero-Guerra, 2018).

\section{Rendimiento académico}

La evaluación diagnóstica, formativa y sumativa se aplican en la enseñanza de la Botánica en el contexto universitario. Desde lo conceptual, la evaluación diagnóstica determina el punto de partida del estudiante y evalúa experiencias, actitudes, hábitos y razonamientos (Bermúdez et al., 2017). En cambio, en la evaluación formativa, el estudiante se considera un sujeto en constante desarrollo al que se le hace un seguimiento paulatino y continuo de su aprendizaje durante el período de duración de la asignatura, el cual tiene carácter inclusivo y es mejorable en el tiempo (Black y Wiliam, 2018). La evaluación formativa es complementaria a la evaluación sumativa, que determina el dominio y la calidad del conocimiento del estudiante de un conjunto de temas (Buchholtz et al., 2018). Esto exige efectuar cambios en el sistema de evaluación de los estudiantes del método tradicional a la evaluación formativa, correlacionada directamente con la preparación integral de nuestros discípulos para la ciudadanía (Guerrero et al., 2017).

Diversos factores contribuyen significativamente al rendimiento académico de los estudiantes de Biología o de especialidades afines, entre los cuales tenemos: (1) la motivación por aprender, el trabajo sistemático en la asignatura, la disponibilidad de libros de textos en las bibliotecas y de equipos de laboratorio (Dinah, 2013); (2) la frecuencia de prácticas de laboratorio, la ejecución de proyectos, y el esfuerzo de los estudiantes (Solar, 2015); (3) la cualificación del profesor y su asistencia para impartir las clases, así como la idoneidad de las computadoras y la reducción del número de estudiantes por aula (Ali et al., 2014); (4) el modo de vida, la institución pública o privada donde efectuaron sus estudios y la presencia/ausencia de obligaciones laborales (Arroyo et al., 2009).

La presente investigación valida la hipótesis que el enfoque pedagógico que elige el docente influye significativamente en el rendimiento académico de los estudiantes. El objetivo general es: evaluar tres modelos pedagógicos para la enseñanza-aprendizaje de la asignatura Botánica General con el propósito de mejorar la calidad de su enseñanza e incentivar la motivación de los estudiantes por esta ciencia. Los siguientes objetivos específicos se evalúan en los estudiantes de primer semestre, Ingeniería Agropecuaria, asignatura de Botánica General: (1) determinar sus estilos de aprendizaje en correspondencia con el género y la edad; (2) determinar sus estrategias de aprendizaje cooperativo, profundo y autónomo en correspondencia con el género y la edad; (3) determinar el efecto del modelo pedagógico en su evaluación formativa, sumativa y final de la asignatura; (4) efectuar una valoración cuantitativa de su percepción en relación a los modelos pedagógicos empleados y las actividades desarrolladas en la asignatura.

\section{MATERIALES Y MÉTODOS}

Esta seccion e estructura en seis sub-secciones: toma de muestra, sílabo de la asignatura botánica general, metodología, instrumentos, variables, análisis estadístico

\section{Toma de muestra}

El estudio se efectúa con los estudiantes que cursaron la asignatura de Botánica General, primer semestre, carrera de Ingeniería Agropecuaria de la Universidad Estatal Amazónica de Ecuador. Del total de 29 estudiantes matriculados, 17 son hombres y 12 son mujeres.

\section{Sílabo de la asignatura Botánica General}

La asignatura Botánica General consta de tres unidades. En la Unidad I "La Célula" se abordan 7 temas y se efectúan 2 prácticas de laboratorio. Mientras que en la Unidad II "Tejidos Vegetales" se imparten 7 temas y cuatro prácticas de laboratorio. En cambio, en la Unidad III se estudian los órganos de las plantas y los sistemas de dispersión de frutos y semillas que ocupan 8 temas y 7 prácticas de laboratorios. Los contenidos tienen una complejidad análoga en todas las unidades. Al inicio de la asignatura la docente explica la metodología a seguir en cada una de las unidades objeto de estudio. 


\section{Metodología}

En la primera unidad - La Célula - se imparten las clases teóricas y las prácticas de laboratorio por el método tradicional. El trabajo por equipos es esencial. Se forman catorce equipos al azar mediante sorteo, trece de 2 estudiantes y uno de 3 integrantes. Los equipos, después de recibir cada clase teórica, trabajan en la resolución de cuestionarios académicos de 8-26 preguntas/tema vía plataforma Moodle. La preparación de los informes de las prácticas de laboratorio también se efectúa por equipos.

En la segunda unidad - Tejidos Vegetales - se aplica un modelo mixto; en las clases teóricas se sigue el método tradicional y en las prácticas de laboratorio se emplea el modelo del aula invertida. Cada estudiante contesta un cuestionario académico de 6-20 preguntas vía plataforma Moodle como parte de su preparación previa a cada práctica de laboratorio. La preparación de los informes de las prácticas de laboratorio también es individual.

En la última unidad - Organografía y Sistemas de Dispersión del Polen, Frutos y Semillas - se imparten las clases teóricas y las prácticas de laboratorio mediante el enfoque de aula invertida, y se incrementa el trabajo individual del estudiante. Se incentiva la participación en las clases teóricas ( 8 en total) mediante las técnicas de taller, seminario, mesa redonda, grupos de discusión, debate a través de preguntas y respuestas, y lluvia de ideas por tarjetas. Cada estudiante contesta cuestionarios de 10 preguntas a través de la plataforma Moodle como parte de su preparación previa a cada práctica de laboratorio.

Para las prácticas de laboratorio de las tres unidades de aprendizaje se entrega una guía detallada con imágenes y esquemas de las observaciones a efectuar al microscopio óptico o al microscopio estereoscópico, y se acompaña de vídeos instructivos de los temas objeto de estudio. El docente al inicio de cada sesión de trabajo de laboratorio y a través de preguntas y respuesta con sus estudiantes realiza el debate introductorio del trabajo a realizar, además de clarificar sus inquietudes y supervisar el desarrollo de la actividad (Rivero-Guerra, 2018). Durante las prácticas de laboratorio se comparten los materiales y los microscopios por pareja.

\section{Instrumentos}

Durante la investigación se emplean 5 instrumentos para la obtención de los datos. Al inicio de la asignatura se aplica el cuestionario Honey-Alonso de Estilos de Aprendizaje (CHAEA) de 80 enunciados (Alonso et al., 1999). Se evalúan cuantitativamente (0, mínimo, al 5, máximo) las estrategias de aprendizaje cooperativo (20 enunciados; Atxurra et al., 2015), aprendizaje profundo (20 enunciados; Gargallo et al., 2006) y aprendizaje autónomo (45 enunciados; López-Aguado, 2010) que aplican los estudiantes en su desempeño en la asignatura. Se mide cuantitativamente (0, mínimo, al 5, máximo) la percepción de los estudiantes en relación a los modelos pedagógicos que se emplean y las actividades que se realizan, a través de un cuestionario de 33 enunciados al finalizar los contenidos teóricos y prácticos de cada una de las unidades de aprendizaje de la asignatura (Tabla 1).

El tiempo de aplicación de cada instrumento oscila de 15 a 20 minutos, los estudiantes acceden de forma voluntaria a colaborar en la investigación. Los cuestionarios son anónimos y durante su ejecución el docente sale del aula. El presidente del paralelo se encarga de recoger los cuestionarios cumplimentados y los entrega al docente. La opinión de cada participante se considera como una unidad independiente operativa para todos los cuestionarios que se aplican y analizan estadísticamente. Los resultados de la investigación se exponen a los estudiantes.

\section{Variables}

El presente estudio considera como variables independientes los métodos de enseñanza que se aplican (tradicional, mixto y de aula invertida), el género (hombre o mujer) y la edad de los estudiantes (18-30 años). Las variables dependientes que se estudian son: los estilos de aprendizaje, las estrategias de aprendizaje cooperativo, profundo y autónomo, las evaluaciones formativa y sumativa (Examen Unidad I, Examen Unidad II, y Examen Unidad III), y la calificación final de la asignatura; además de la percepción de los estudiantes en relación a los modelos pedagógicos que se emplean y las actividades que se realizan. La máxima calificación para la evaluación formativa, sumativa y final es de 10 puntos, y el aprobado se alcanza con 7 puntos. 
Tabla 1: Matriz con el cuestionario para medir cuantitativamente ( 0 , mínimo, al 5, máximo) la percepción de los estudiantes en relación al modelo pedagógico y las actividades que se efectúan en la asignatura Botánica General.

1. Puntúe su nivel de preparación previa para cada una de las clases teóricas

2. Puntúe su nivel de preparación previa para cada una de las prácticas de laboratorio

3. Puntúe la explicación ofrecida por el docente en la clase teórica sobre la programación, los objetivos a lograr, las actividades a desarrollar, la metodología a emplear, la bibliografía, la distribución del tiempo, el sistema de evaluación y las cuestiones técnicas para el manejo de la Plataforma Moodle

4. Puntúe la calidad científica y didáctico-educativa de los contenidos abordados y de los objetivos propuestos

5. Puntúe la originalidad, claridad y compresión del material didáctico proporcionado por el docente a través de la Plataforma Moodle

6. Puntúe la enseñanza y el desempeño del docente en relación a la manipulación de los materiales, instrumental y equipos en el laboratorio que favorecen su trabajo autónomo

7. Puntúe el desempeño del docente al supervisar el desarrollo de las prácticas de laboratorio

8. Puntúe su habilidad en identificar y delimitar sus debilidades y carencias al estudiar los contenidos de la unidad de aprendizaje

9. Puntúe el uso de la Plataforma Moodle para facilitar el aprendizaje

10. Puntúe las posibilidades de acceso al material biológico propuesto para las prácticas de laboratorio

11. Puntúe el nivel de claridad, comprensibilidad y facilidad de evaluar los objetivos propuestos en las clases teóricas y en las prácticas de laboratorio

12. Puntúe la utilidad de los vídeos de apoyo y las grabaciones en el aprendizaje de los contenidos

13. Puntúe su nivel de motivación y participación en clase

14. Puntúe la adecuación de la bibliografía proporcionada por el docente a los contenidos de cada tema

15. Puntué el tamaño y estado de las aulas en proporcionar un ambiente agradable y propicio para el aprendizaje

16. Puntúe su desempeño al aplicar los conocimientos teóricos recibidos en clase a las prácticas de laboratorio
17. Puntúe el logro de los objetivos propuestos en el tiempo designado para las clases teóricas y prácticas de laboratorio

18. Puntúe su grado de aprendizaje activo en el laboratorio

19. Puntúe su grado de aprendizaje autónomo en el laboratorio después de haber clarificado todas sus inquietudes con el docente

20. Puntúe las estrategias y actividades creativas y variadas desplegadas por el docente para facilitar el desarrollo de competencias y dinamizar el aprendizaje

21. Puntúe sus habilidades en plantearse y resolver problemas mediante la búsqueda de nuevas soluciones a cuestiones específicas de esta unidad de aprendizaje

22. Puntúe la utilidad de los conocimientos adquiridos en esta unidad de aprendizaje para su desempeño en otras asignaturas de la carrera

23. Puntúe el nivel de organización y estructura de las clases teóricas y de las prácticas de laboratorio

24. Puntúe el estado de los microscopios y de los equipos de laboratorio

25. Puntúe si las actividades realizadas fomentan el trabajo cooperativo estudiante-estudiante y facilitan la adquisición de conocimientos

26. Puntúe su nivel de aprendizaje autónomo fuera del aula

27. Puntúe la relación y comunicación estudiantedocente

28. Puntúe si la distribución teórico-práctica (proporción entre conocimientos teóricos y prácticos) son suficiente para alcanzar los objetivos propuestos

29. Puntúe la necesidad de incrementar el número de prácticas de laboratorio en esta unidad de aprendizaje

30. Puntúe su grado de satisfacción con los contenidos y protocolos de las prácticas de laboratorio realizadas

31. Puntúe su percepción general sobre el método empleado para impartir los temas de esta unidad de aprendizaje

32. Puntúe su percepción general sobre el método pedagógico empleado en esta unidad de aprendizaje en relación a su rendimiento académico

33. Puntúe su nivel de satisfacción con la calificación otorgada por el docente a su desempeño

\section{Análisis estadístico}

En la estadística descriptiva se determina el rango de variación, la media y la desviación estándar de las variables objeto de estudio. El coeficiente de variación no se determina al ser la variación de la desviación estándar baja, al presentar los datos poca dispersión. Se aplica la técnica de ANOVA para analizar la variación de la edad de los estudiantes matriculados en la asignatura Botánica General. Se analiza la partición de la varianza mediante la técnica de MANOVA: (1) entre géneros (hombre y mujer), a nivel de edad y en la interacción género $x$ edad de los estilos de aprendizaje y de las estrategias de aprendizaje cooperativo, profundo y autónomo; y (2) entre los métodos que se aplican, entre géneros, en relación a la edad $\mathrm{y}$ en la interacción método $\mathrm{x}$ género, método $\mathrm{x}$ edad, género $\mathrm{x}$ edad, método $\mathrm{x}$ género $\mathrm{x}$ edad de las evaluaciones formativa, sumativa y final de los estudiantes.

Se aplica la técnica de Análisis de Componentes Principales para explorar la estructura de correlación entre las variables y efectuar una reducción de las variables objeto de estudio, las cuales: (A) definen las estrategias de aprendizaje de los estudiantes y sus calificaciones formativa, sumativa y final, y (B) describen la percepción general de los estudiantes en relación a la metodología que se emplea y establece la 
importancia relativa de cada variable en la disimilitud entre los estudiantes. El coeficiente de correlación se considera alto para $r \geq 0,75$, moderado cuando $0,50 \leq r<0,75$, y bajo cuando $r<0,50$. La variación de los factores extraídos se analiza mediante la técnica de MANOVA: (1) entre géneros, en relación a la edad, y en la interacción género x edad para "(A)" y (2) entre los métodos que se aplican, entre géneros, en relación a la edad, $\mathrm{y}$ en la interacción método $\mathrm{x}$ género, método $\mathrm{x}$ edad, género $\mathrm{x}$ edad, método $\mathrm{x}$ género $\mathrm{x}$ edad para "(B)". El análisis de conglomerados se efectúa por el método de agrupación del vecino más cercano y emplea como intervalo la distancia Euclídea al cuadrado para representar las relaciones entre los estudiantes respecto al método pedagógico propuesto en cada una de las unidades de aprendizaje.

Las técnicas se aplican después de garantizar el cumplimiento de los requisitos de distribución de datos para (1) la normalidad multivariante (MANOVA) o univariante (ANOVA) mediante el contraste de Kolmogorov-Smirnov y Shapiro-Wilk, respectivamente; (2) la homogeneidad de la varianza mediante el contraste de Barlett-Box en los modelos multivariantes, la prueba Kaiser-Meyer-Olkin (KMO) previo al análisis de los componentes principales y la prueba de Levene en el modelos univariantes (Dytham 2003). La presencia de valores raros o valores atípicos se detecta gráficamente porque el MANOVA es sensible a ellos. Los valores raros o atípicos se observan para los datos de las variables calificaciones formativas, sumativa y final, las cuales se transforman mediante la raíz cuadrada antes del análisis para aumentar su homogeneidad de varianza, mientras que la comparación con los resultados originales indica diferencias menores. El resto de las variables que se analizan cumplen con los requisitos de normalidad y homogeneidad de varianzas.

Se emplean los paquetes estadísticos STATISTICA versión 7.0 (StatSoft, Tulsa, Oklahoma) y SPSS versión 20.0 (SPSS, Chicago, Illinois). Los resultados de cada uno de los análisis que se aplican se consideran significativos si la probabilidad de la hipótesis nula fue menor que 0,05 . Todos los resultados numéricos se expresan con dos decimales para incrementar la precisión de cada valor o medida que se plasma.

\section{RESULTADOS}

Los estudiantes de primer semestre de Ingeniería Agropecuaria presentan una edad comprendida entre 18 y 30 años, con una edad media de 20,52 años (Tabla 2). El estudio sobre los estilos de aprendizaje indica que los estudiantes son en mayoría reflexivos (Tabla 3), con un equilibrio entre el número de estudiantes pragmáticos, teóricos y activos. Los hombres no presentan un estilo de aprendizaje preferente al existir estabilidad entre los estilos reflexivo, pragmático y teórico. Las mujeres fueron pluralmente reflexivas y el resto no presenta un estilo de aprendizaje preferencial con valores análogos para la combinación pragmático-activo-teórico. No se aprecian diferencias estadísticamente significativas entre géneros ( $\lambda$ de Wilks $=0,70 ; F_{4,17}=1,78 ; p>0,05$ ), a nivel de la edad ( $\lambda$ de Wilks $=0,41 ; F_{28 ; 62,72=0,63 ; p>0,05}$ ) y en la interacción género $x$ edad $\left(\lambda\right.$ de Wilks $\left.=0,90 ; F_{4,17}=0,45 ; p>0,05\right)$ de los estudiantes.

Tabla 2: Variación no significativa de la edad de los estudiantes de primer semestre de Ingeniería Agropecuaria, asignatura Botánica General $\left(F_{1,28}=0,17 ; p>0,05\right)$.

\begin{tabular}{|c|c|c|c|c|c|c|c|}
\hline \multicolumn{4}{|c|}{} & \multicolumn{2}{c|}{ Hombre $(N=17)$} & \multicolumn{2}{c|}{ Mujer $(N=12)$} \\
\hline Variable & $N$ & Rango & Media $\pm D e$ & Rango & Media $\pm D e$ & Rango & Media $\pm D e$ \\
\hline Edad & 29 & $18-30$ & $20,52 \pm 2,99$ & $19-23$ & $20,53 \pm 1,37$ & $18-30$ & $20,50 \pm 4,48$ \\
\hline
\end{tabular}

El aprendizaje cooperativo de los estudiantes alcanza valores en el rango de 2,92 a 4,25 puntos de un máximo de 5 (Tabla 4). Ambos sexos consiguen puntuaciones superiores a 4 puntos en relación a: "en esta clase los estudiantes reciben las calificaciones que se merecen, ni más, ni menos (P3)", "el profesor nos da pautas para resolver los conflictos que pueden surgir en el grupo (P7)", y "en esta asignatura, mejorar las habilidades para relacionarnos con los demás, es un objetivo a lograr (P10)" para los hombres y "en esta asignatura se promueve el respeto en las relaciones grupales (P17)" y "en el grupo valoramos la actuación de cada uno de nosotros y damos orientaciones para mejorar (P20) para las mujeres. Las puntuaciones inferiores a 3 puntos se obtienen para "el profesor nos ayuda a identificar y definir las dificultades del trabajo grupal (P4)" según el criterio de las mujeres.

No imperan diferencias estadísticamente significativas entre géneros $\left(\lambda\right.$ de Wilks $\left.=0,70 ; F_{4,17}=1,79 ; p>0,05\right)$; lo contrario ocurre para la edad $\left(\lambda\right.$ de Wilks $\left.=2,95 \times 10^{-7} ; F_{114 ; 18,84}=2,08 ; p<0,05\right)$ y en la interacción género $x$ edad $\left(\lambda\right.$ de Wilks $\left.=0,05 ; F_{19,2}=21,94 ; p<0,05\right)$ de los estudiantes. El análisis univariante de la varianza revela que los enunciados $\mathrm{P} 1\left(\mathrm{~F}_{1,20}=4,35 ; \mathrm{p}<0,05\right)$ y $\mathrm{P} 10\left(\mathrm{~F}_{6,20}=3,30 ; \mathrm{p}<0,05\right)$ exhiben significación estadística a nivel de edad y en la interacción género $x$ edad de los estudiantes respectivamente. 
Tabla 3: Estilos de aprendizaje de los estudiantes de primer semestre de Ing. Agropecuaria, asignatura Botánica General.

\begin{tabular}{|l|c|c|c|c|c|c|c|}
\hline \multicolumn{4}{|c|}{} & \multicolumn{2}{c|}{ Hombre $(N=17)$} & \multicolumn{2}{c|}{ Mujer $(N=12)$} \\
\hline Variable & $N$ & Rango & Media $\pm D e$ & Rango & Media $\pm D e$ & Rango & Media $\pm D e$ \\
\hline Activo & 29 & $7-16$ & $11,07 \pm 2,67$ & $7-16$ & $10,56 \pm 2,45$ & $7-16$ & $11,83 \pm 2,91$ \\
\hline Reflexivo & 29 & $8-18$ & $13,33 \pm 2,64$ & $9-18$ & $12,78 \pm 2,62$ & $8-17$ & $14,17 \pm 2,55$ \\
\hline Teórico & 29 & $6-17$ & $11,93 \pm 3,08$ & $6-17$ & $12,39 \pm 2,93$ & $6-16$ & $11,25 \pm 3,31$ \\
\hline Pragmático & 29 & $8-18$ & $12,57 \pm 2,51$ & $10-18$ & $12,72 \pm 2,02$ & $8-18$ & $12,33 \pm 3,20$ \\
\hline
\end{tabular}

Tabla 4: Estadística descriptiva de los cuestionarios de estrategias de aprendizaje cooperativo, profundo y autónomo de los estudiantes de primer semestre de Ingeniería Agropecuaria, asignatura Botánica General.

\begin{tabular}{|l|c|c|c|c|c|c|c|c|}
\hline & \multicolumn{4}{|c|}{} & \multicolumn{2}{c|}{ Hombre $(N=17)$} & \multicolumn{2}{c|}{ Mujer (N=12) } \\
\hline Aprendizaje & Variable & $N$ & Rango & Media \pm De & Rango & Media $\pm D e$ & Rango & Media \pm De \\
\hline Cooperativo & P1-P20 & 29 & $3,48-4,03$ & $3,76 \pm 1,25$ & $3,65-4,24$ & $3,86 \pm 1,09$ & $2,92-4,25$ & $3,63 \pm 1,43$ \\
\hline Profundo & P1-P21 & 29 & $3,17-3,93$ & $3,62 \pm 1,21$ & $3,47-4,24$ & $3,81 \pm 1,04$ & $2,75-4,00$ & $3,37 \pm 1,39$ \\
\hline Autónomo & P1-P45 & 29 & $3,00-3,97$ & $3,58 \pm 1,04$ & $3,06-4,06$ & $3,39 \pm 1,01$ & $2,50-4,17$ & $3,43 \pm 1,09$ \\
\hline
\end{tabular}

En términos de aprendizaje profundo se obtienen puntuaciones en el rango de 2,75 a 4,00 (Tabla 4). Ambos géneros alcanzan puntuaciones superiores a 4 puntos para: "encuentro que a veces estudiar me proporciona un sentimiento de profunda satisfacción personal (P1)" respecto a las mujeres; "cuando estudio algo, tengo que trabajarlo bastante para formarme una opinión personal al respecto, y así quedarme satisfecho (P2)", "cualquier tema puede ser interesante cuando se profundiza en su contenido (P5)", "encuentro interesante la mayoría de los temas nuevos y a menudo dedico tiempo extra a ampliarlos buscando información adicional (P6)" y "me hago preguntas sobre aquellos temas que considero importantes hasta que los comprendo totalmente (P10)" concerniente a los hombres. Se alcanzan valores inferiores a 3 puntos en relación a: "realmente sólo estudio los apuntes y lo que se señala en clase; entiendo que buscar información complementaria por mi cuenta es una pérdida de tiempo (P4)", "aprendo algunas cosas mecánicamente, repitiéndolas una y otra vez hasta que las sé de memoria aunque no las entienda" (P8) y "estudiar temas académicos puede ser a veces tan atractivo como leer una buena novela o ver una buena película (P9)" en las mujeres. No se aprecian diferencias estadísticamente significativas entre géneros ( $\lambda$ de Wilks=0,30; $\left.F_{19,2}=3,44 ; p>0,05\right)$, a nivel de edad $\left(\lambda\right.$ de Wilks $=5,00 \times 10^{-5} ; F_{114 ; 18,84}=1,19 ; p>0,05$ ) $y$ en la interacción género $x$ edad $\left(\lambda\right.$ de Wilks $\left.=0,21 ; F_{19,2}=4,79 ; p>0,05\right)$ de los estudiantes.

Los estudiantes muestran puntuaciones en el rango de 2,50 a 4,17 en relación a las estrategias de aprendizaje autónomo (Tabla 4). Ambos géneros presentan puntuaciones superiores a 4 puntos para: "evalúo el proceso de aprendizaje final (P17)" y "para preparar el examen me baso principalmente en los aspectos que el profesor marca como importante (P35)" respecto a los hombres y "tomo nota de las respuestas del profesor (P1)", "realizo un mapa conceptual con los conceptos más importantes de cada apartado (P11)", "preparo los exámenes teniendo en cuenta todo el material, no sólo los apuntes (P25)", "confecciono un resumen de cada tema (P32), y trabajo en colaboración para resolver un problema o investigar algo (P37)" en relación a las mujeres. Las puntuaciones inferiores a 3 puntos se obtienen para "me organizo con los compañeros para pedir libros en la biblioteca (P23)", "cuando descubro aportaciones nuevas en documentos complementarios a la bibliografía recomendada, lo comparto con los compañeros (P24)", "consulto con los compañeros las dudas que se me plantean en el estudio del tema (P27)", "reparto con algunos compañeros los libros de la bibliografía básica, elaborando resúmenes de cada uno de ellos, para compartirlos (P31)" y "sigo, aprovecho y participo en clase (P41)" en el caso de las mujeres. No se observan diferencias estadísticamente significativas entre géneros ( $\lambda$ de Wilks $\left.=0,09 ; F_{20,1}=5,72 ; p>0,05\right)$, a nivel de edad $\left(\lambda\right.$ de Wilks $\left.=7,00 \times 10^{-5} ; F_{120 ; 13,24}=0,75 ; p>0,05\right)$ y en la interacción género $x$ edad $(\lambda$ de Wilks $\left.=0,23 ; F_{20,1}=1,70 ; p>0,05\right)$ de los estudiantes.

La calificación media de las evaluaciones formativas, correspondientes al enfoque de aula invertida, es significativamente superior en relación a los dos métodos previos que se aplican (Tabla 5). La puntuación media en el Examen de la Unidad I, Método I (tradicional) (5,31 $\pm 0,64$; rango: 4-7; $\mathrm{N}=29)$ y en el Examen de la Unidad II, Método II (mixto) (5,0 $\pm 1,51$; rango: 2-8; $\mathrm{N}=29)$ fue por debajo del aprobado. Se logran puntuaciones satisfactorias $(8,97 \pm 1,15$; rango: $5-10 ; \mathrm{N}=29$ ) en el Examen de la Unidad III, Método III (aula invertida). Un total de 11 estudiantes acuden al examen supletorio y obtienen una calificación media provechosa $(8,10 \pm 1,90$; rango: $2,5-9,8 ; \mathrm{N}=11)$. En general, el $93,10 \%$ de los estudiantes aprueban de forma satisfactoria la asignatura, en concepto de reprobados quedaron dos estudiantes. 
Tabla 5: Resumen de las calificaciones de la evaluación formativa de los estudiantes de primer semestre de Ingeniería Agropecuaria, asignatura Botánica General en relación a la metodología que se aplica. Método I (tradicional), Método II (mixto) y Método III (aula invertida).

\begin{tabular}{|l|c|c|c|c|c|c|}
\hline & \multicolumn{2}{|c|}{$\begin{array}{c}\text { Formativa } \\
\text { Método I }(N=29)\end{array}$} & \multicolumn{2}{c|}{$\begin{array}{c}\text { Formativa } \\
\text { Método Ii }(N=29)\end{array}$} & \multicolumn{2}{c|}{$\begin{array}{c}\text { Formativa } \\
(N=29)\end{array}$} \\
\hline Variable & Rango & Media \pm De & Rango & Media \pm De & Rango & Media \pm De \\
\hline Calificaciones & $4,1-8,7$ & $7,41 \pm 1,08$ & $5,3-8,6$ & $7,29 \pm 0,91$ & $8,0-10,0$ & $9,21 \pm 0,72$ \\
\hline
\end{tabular}

El análisis multivariante de la varianza denota diferencias significativas en las calificaciones obtenidas en relación a la metodología que se aplica ( $\lambda$ de Wilks $\left.=0,22 ; F_{4,118}=32,78 ; p<0,05\right)$. Respecto al género $(\lambda$ de Wilks= 0,99; $\left.F_{2,59}=0,06 ; p>0,05\right)$, la edad ( $\lambda$ de Wilks=0,79; $\left.F_{12,118}=1,19 ; p>0,05\right)$, el método $x$ género $(\lambda$ de Wilks $\left.=0,96 ; F_{4,118}=0,61 ; p>0,05\right)$, el método $x$ edad $\left(\lambda\right.$ de Wilks $\left.=0,70 ; F_{24,118}=0,94 ; p>0,05\right)$, el género $x$ edad $\left(\lambda\right.$ de Wilks $\left.=0,99 ; F_{2,59}=0,03 ; p>0,05\right)$ y el método $x$ género $x$ edad $\left(\lambda\right.$ de Wilks $=0,92 ; F_{4,118}=1,19$; $p>0,05)$ no se obtienen resultados estadísticamente significativos. El análisis univariante de la varianza demuestra que la calificación formativa $\left(F_{2,60}=23,68 ; p<0,00001\right)$ y los exámenes $\left(F_{2,60}=75,05 ; p<0,00001\right)$ son estadísticamente significativos en relación a la metodología aplicada; que se confirma a través del análisis de conglomerados (Fig. 1). En este último se muestra que el método de enseñanza de aula invertida está significativamente diferenciado de los otros dos métodos en relación al rendimiento académico de los estudiantes (Fig. 1).

El análisis de los Componentes Principales extrae tres componentes: componente 1 (autovalor=3,16; porcentaje de la varianza=35,14), componente 2 (autovalor=2,06; porcentaje de la varianza=22,95) y componente 3 (autovalor $=1,11$; porcentaje de la varianza $=12,31$. La prueba de esfericidad de Bartlett señala significación estadística $\left(X^{2}=121,63 ; g l=36 ; p<0,00001\right)$. La matriz de componentes rotados por el método varimax (Fig. 2) refleja que: (1) existe una fuerte correlación entre los estilos de aprendizaje y el componente 1 (aprendizaje profundo $r=0,94$; aprendizaje cooperativo $r=0,91$; aprendizaje autónomo $r=$ $0,90)$; (2) que hay una correlación moderada entre la calificación formativa $(r=0,73)$, la calificación final de la asignatura $(r=0,72)$, la calificación del Examen de la Unidad II $(r=0,66)$, los estilos de aprendizaje $(r=-0,60)$ y la calificación del Examen de la Unidad III $(r=0,57)$ con el componente 2; donde los estilos de aprendizaje marcan una correlación con signo negativo con este componente; (3) la calificación del Examen de la Unidad I $(r=0,83)$ registra una fuerte correlación con el componente 3.

El análisis de los Componentes Principales extrae tres componentes: componente 1 (autovalor=3,16; porcentaje de la varianza $=35,14$ ), componente 2 (autovalor=2,06; porcentaje de la varianza=22,95) y componente 3 (autovalor=1,11; porcentaje de la varianza=12,31). La prueba de esfericidad de Bartlett señala significación estadística $\left(X^{2}=121,63 ; g l=36 ; p<0,00001\right)$. La matriz de componentes rotados por el método varimax (Fig. 2) refleja que: (1) existe una fuerte correlación entre los estilos de aprendizaje y el componente 1 (aprendizaje profundo $r=0,94$; aprendizaje cooperativo $r=0,91$; aprendizaje autónomo $r=$ $0,90)$; (2) que hay una correlación moderada entre la calificación formativa $(r=0,73)$, la calificación final de la asignatura $(r=0,72)$, la calificación del Examen de la Unidad II $(r=0,66)$, los estilos de aprendizaje $(r=-0,60)$ y la calificación del Examen de la Unidad III $(r=0,57)$ con el componente 2; donde los estilos de aprendizaje marcan una correlación con signo negativo con este componente; (3) la calificación del Examen de la Unidad I $(r=0,83)$ registra una fuerte correlación con el componente 3 .

El análisis factorial completo demuestra que no existe variación significativa para los componentes factoriales $1-3$ a nivel de género ( $\lambda$ de Wilks $\left.=0,69 ; F_{3,18}=2,64 ; p>0,05\right)$ y en la interacción género $x$ edad $(\lambda$ de Wilks=0,83; $F_{3,18}=1,26 ; p>0,05$ ); lo opuesto ocurre en relación a la edad ( $\lambda$ de Wilks=0,16; $F_{18 ; 51,39=2,62 ;}$ $\mathrm{p}<0,05)$. El análisis univariante de la varianza prueba que existe significación estadística para el componente factorial 1 respecto a la edad de los estudiantes $\left(F_{6,20}=5,46 ; p<0,05\right)$. Los estudiantes valoraron satisfactoriamente de forma cuantitativa la metodología aplicada en cada unidad de aprendizaje de la asignatura (Tabla 6). El análisis de los componentes principales extrae dos componentes. El componente 1 (autovalor $=10,54$; porcentaje de la varianza $=31,97$ ) muestra una correlación moderada con los enunciados P6 $(r=0,74)$ y P13 $(r=0,70)$. Cuestión análoga ocurre para los enunciados P21 $(r=0,72)$ y P25 $(r=0,70)$ en relación al componente 2 (autovalor=2,15; porcentaje de la varianza $=6,50$ ).

El análisis de la varianza de los componentes extraídos por el modelo muestra que el criterio de los estudiantes se mantuvo estable durante todo el semestre respecto a: (1) la metodología aplicada ( $\lambda$ de Wilks=0,87; $F_{4,106}=1,87 ; p>0,05$ ), (2) el género ( $\lambda$ de Wilks=0,92; $\left.F_{2,53}=2,22 ; p>0,05\right)$, (3) la edad ( $\lambda$ de Wilks $=0,85 ; F_{14,106}=0,66 ; p>0,05$ ), (4) el método $x$ género ( $\lambda$ de Wilks $=0,98 ; F_{4,106}=0,33 ; p>0,05$ ), (5) el método $x$ edad $\left(\lambda\right.$ de Wilks $\left.=0,86 ; F_{28,106}=0,29 ; p>0,05\right)$, (6) el género $x$ edad $\left(\lambda\right.$ de Wilks $=0,95 ; F_{4,106}=0,70$; $p>0,05)$, y (7) el método $x$ género $x$ edad $\left(\lambda\right.$ de Wilks $\left.=0,85 ; F_{8,106}=1,87 ; p>0,05\right)$. 


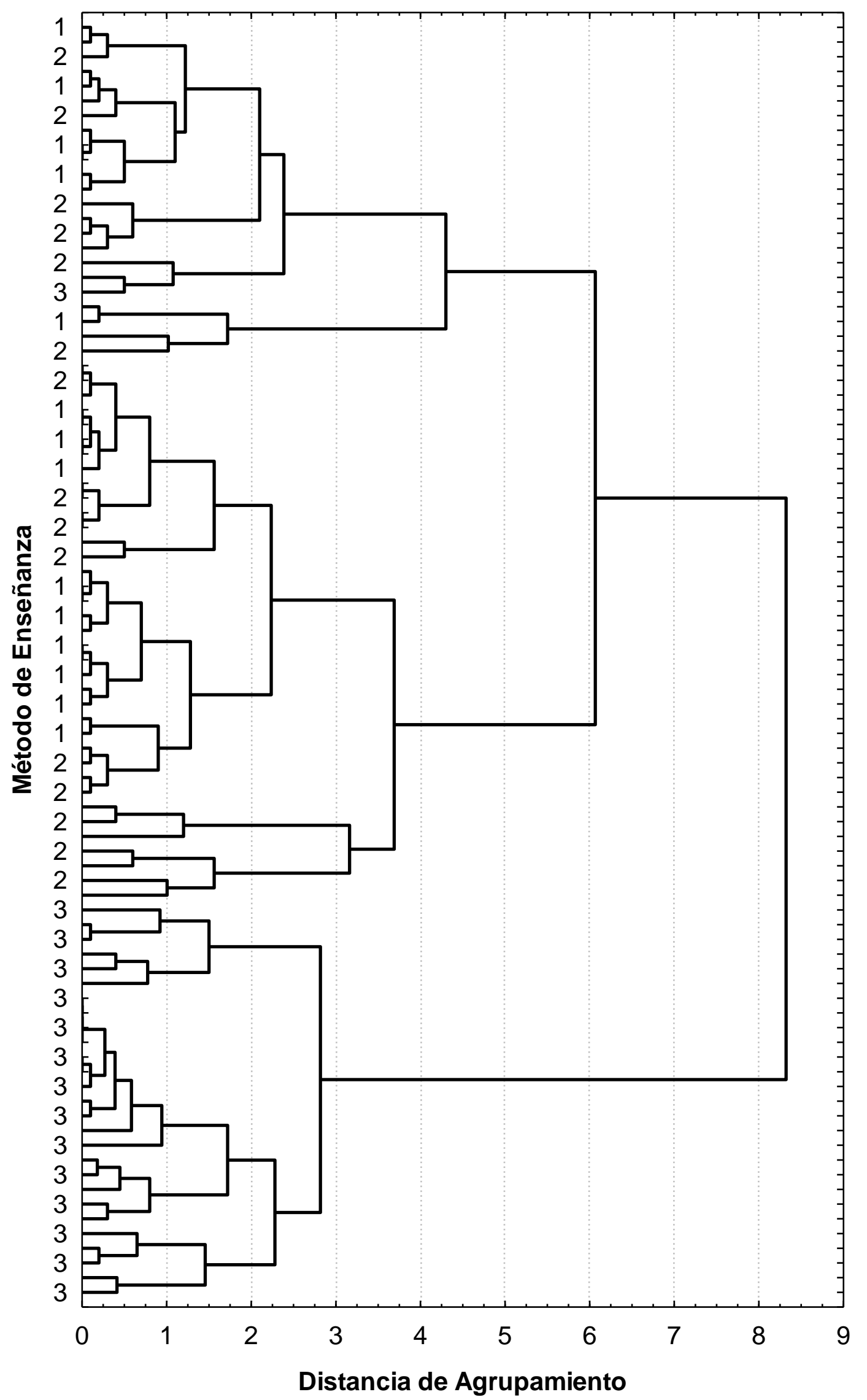

Fig.1: Conglomerado construido en base al rendimiento académico de los estudiantes según el método de enseñanza. Método: tradicional (1), mixto (2) y aula invertida (3). 


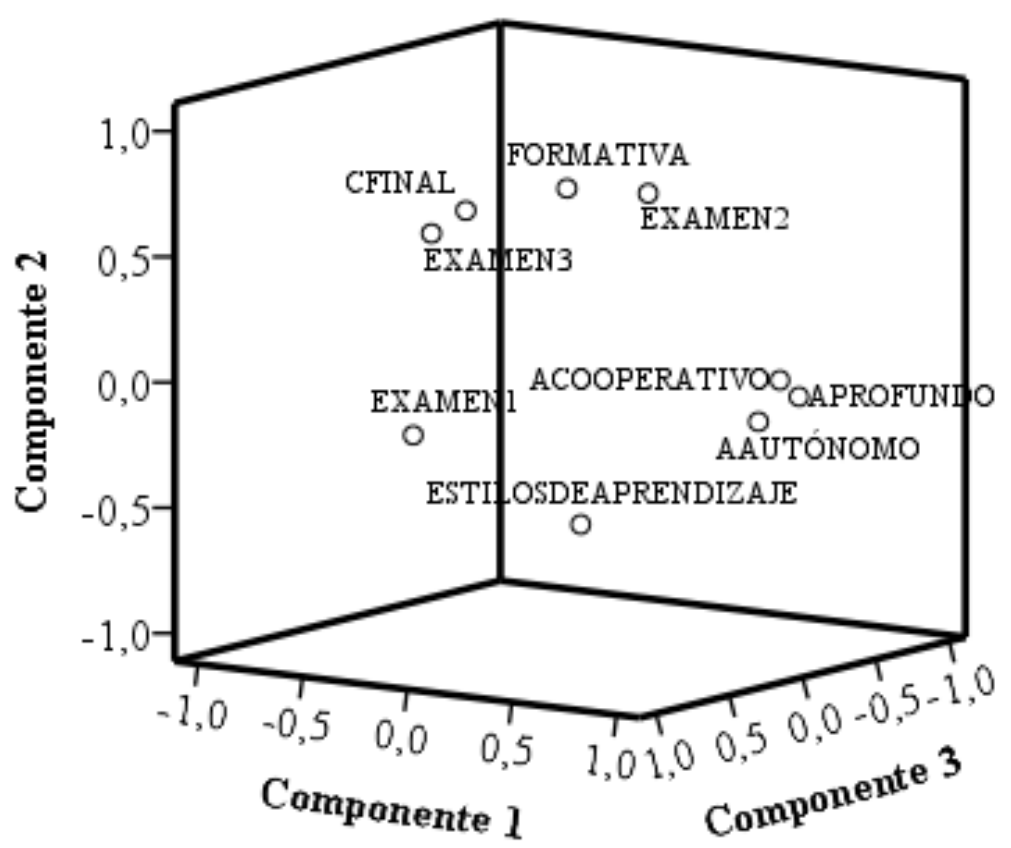

Fig. 2: Componentes principales en espacio rotado.

Tabla 6: Percepción cuantitativa de los estudiantes de primer semestre, Ingeniería Agropecuaria respecto a los tres métodos de enseñanza empleados en las Unidades I-III, asignatura Botánica General. Método: tradicional (I), mixto (II) y aula invertida (III).

\begin{tabular}{|c|c|c|c|c|c|c|}
\hline & \multicolumn{2}{|c|}{$\begin{array}{c}\text { Unidad I } \\
\text { Método I } \\
(N=29)\end{array}$} & \multicolumn{2}{c|}{$\begin{array}{c}\text { Unidad li } \\
\text { Método li } \\
(N=29)\end{array}$} & \multicolumn{2}{c|}{$\begin{array}{c}\text { Unidad lii } \\
\text { Método lii } \\
\text { (N=29) }\end{array}$} \\
\hline Variable & Rango & Media \pm De & Rango & Media \pm De & Rango & Media \pm De \\
\hline P1-P33 & $2,03-4,28$ & $3,74 \pm 0,90$ & $2,69-3,86$ & $3,61 \pm 0,93$ & $3,14-3,90$ & $3,63 \pm 0,95$ \\
\hline
\end{tabular}

\section{DISCUSIÓN}

Los estilos de aprendizaje se relacionan con las teorías de enseñanza. La enseñanza tradicional se relaciona con el estilo de aprendizaje activo; la enseñanza expresiva o activa se correlaciona con el modelo teórico, mientras que la enseñanza emancipadora o crítica se vincula con el estilo pragmático, en cambio, el estilo reflexivo no se relaciona con ninguna teoría de enseñanza (Pacheco y Maldonado, 2017). El estilo de aprendizaje predominante de los estudiantes de primer semestre de Ingeniería Agropecuaria que cursaron la asignatura Botánica General es el reflexivo, con preferencia por la aplicación de las ideas. Esto es muy positivo para la asignatura de Botánica General al combinar la conceptualización, la experimentación activa y su aplicación práctica. En esta asignatura los contenidos se organizan y estructuran para que los estudiantes resuelvan problemas específicos mediante el razonamiento hipotético-deductivo que favorece y fortalece el proceso de adquisición de conocimientos; además de promover una aptitud activa y reflexiva ante el aprendizaje y durante la repetición de situaciones de aprendizaje.

En esta materia los estudiantes ejercitan su capacidad de observación y análisis de muestras de algas, hongos y plantas tanto en el laboratorio como en el campo, además de recopilar información para el desarrollo de trabajos y/o investigaciones, así como optimizar su capacidad de síntesis, análisis y reflexión. La asignatura estimula el desarrollo del estilo pragmático y activo al ofrecerle al estudiante la posibilidad de realizar un conjunto de prácticas de laboratorio que lo acercan a la realidad objetiva y permite establecer nexos entre los procesos y fenómenos de la naturaleza con el objetivo de abordarlos desde una perspectiva evolutiva. Además, los estudiantes interactúan y responden de forma variada a los distintos ambientes de aprendizaje a través de una combinación de estilos que se correlacionan con sus rasgos cognitivos, afectivos y fisiológicos (Suazo, 2007); cuya motivación está influenciada por las estrategias pedagógicas y el carácter innovador del docente (Silva et al., 2018). 


\section{Estrategias de aprendizaje cooperativo, profundo y autónomo}

El aprendizaje cooperativo constituye un salto hacia la creatividad, perfeccionamiento, transformación e innovación en el sistema educativo (Florence, 2017). En la asignatura Botánica General los estudiantes desarrollan competencias transversales genéricas y específicas, esenciales en el proceso de formación de futuros egresados de las universidades. La presente experiencia educativa permite valorar el intercambio y la construcción colectiva de conocimiento útil y significativo en los estudiantes de Ingeniería Agropecuaria. El trabajo en equipo durante la asignatura potencia las relaciones interpersonales, mejora su capacidad de organización y de liderazgo, además de promover la comunicación y la discusión. Las prácticas de laboratorio permiten a los estudiantes de Ingeniería Agropecuaria demostrar su capacidad de aprender y de aplicar la teoría a la práctica de forma autónoma o en equipo, además de adaptarse a nuevas situaciones. El aprendizaje cooperativo favorece las habilidades cognitivas y el pensamiento reflexivo en las ciencias (Morales-Maure et al., 2018) y un incremento en el uso de la tecnología potencia el interés de los estudiantes y su proceso de comprensión (Rodríguez et al., 2017). En relación a las competencias cognitivas los estudiantes de Ingeniería Agropecuaria adquirieron conocimientos que les permiten (1) explicar y diferenciar la estructura de la célula eucariota vegetal, sus organelos y funciones, (2) diferenciar y explicar la estructura y función de los tejidos y órganos de las plantas, y (3) explicar los mecanismos de dispersión del polen, frutos y semillas.

Entre las competencias procedimentales o instrumentales que logran los estudiantes tenemos: (1) manipulan correctamente los microscopios óptimo y esteroscópico, (2) aplican las técnicas adecuadas para observar la anatomía interna e histología de los órganos vegetativos, (3) aplican las técnicas de tinción de la célula vegetal, (4) identifican la célula, organelos celulares e inclusiones citoplasmáticas, (4) identifican tejidos y órganos de las plantas en el campo y en el laboratorio, (5) manejan la clasificación de las diversidad de raíces, tallos, hojas, inflorescencias, flores, frutos y semillas, (6) explican la importancia de las diversidad de plantas del país y su conservación. En términos de competencias actitudinales los estudiantes alcanzan conocimientos básicos para el ejercicio futuro de la profesión con respeto al medio ambiente, con compromiso ético y con una actitud crítica y autocrítica. Además de habilidades de investigación, de relacionar la materia con otras disciplinas; así como de análisis, síntesis e interpretación de información proveniente de diversas fuentes. La plataforma virtual Moodle es un instrumento para el desarrollo de competencias en los estudiantes, de amplia utilidad para la interacción y comunicación entre profesorestudiante y estudiante-estudiante en la asignatura de Botánica. Durante el transcurso de la asignatura, y con el apoyo de la plataforma Moodle, se midió el cumplimiento de las obligaciones académicas; la consulta a bibliotecas virtuales, portales de interés académico-técnico-científico y al material didáctico que proporciona el docente; la respuesta a encuestas, cuestionarios y exámenes; la participación en foros; el liderazgo, el trabajo individual y en equipo de los estudiantes.

El trabajo autónomo es uno de los pilares de la formación por competencias (Shelton-Strong, 2017) y la autoregulación y el éxito del aprendizaje miden la autoeficiencia (Müller y Seufert, 2018). En este sentido, los resultados de la presente investigación indican que las estrategias de ampliación, colaboración, conceptualización, planificación, preparación para los exámenes y participación de los estudiantes de Ingeniería Agropecuaria son insipientes. Los cuales no muestran sólidas estrategias de aprendizaje cooperativo, profundo y autónomo, habilidades no adquiridas en niveles previos; cuya concepción se enmarca en estudiar para aprobar, lo cual se evidencia en las evaluaciones frecuentes y en los exámenes parciales, aunque el aprendizaje duradero y funcional se logra a base de esfuerzo y dedicación. Los estudiantes no efectúan una planificación óptima y estratégica del estudio, del proceso de aprendizaje y de las actividades académicas que tienen que realizar, con escaso monitoreo. Compensa que los estudiantes y docentes reflexionen sobre las formas de aprendizaje y de los procesos cognitivos que conllevan a facilitar la tarea, a tomar las decisiones acertadas, y a reconocer sus habilidades, preferencias, dificultades y carencias; las cuales impulsan la mejora continua de las acciones y ayudan a crear o construir una identidad cognitiva propia en los estudiantes.

\section{Rendimiento académico}

En la asignatura Botánica General se aplicaron tres modelos de enseñanza (tradicional, mixta y de aula invertida), con contenidos de una complejidad análogo en cada uno de los métodos que se emplearon, en la que se alcanza un $93,10 \%$ de aprobados. La metodología de aula invertida tiene un impacto significativo y provechoso en el rendimiento académico; resultado que se apoya en investigaciones efectuadas en otras materias (Sabater-Mateu et al., 2017), aunque puede no existir efecto significativo de este modelo sobre las calificaciones de los estudiantes (Durán et al., 2017). No obstante, el nivel de satisfacción de los estudiantes es estable durante todo el semestre al no existir significación estadística entre los modelos de enseñanza. Se enfatiza en la repercusión positiva de las prácticas de laboratorio en la calificación general de los estudiantes. En el presente estudio la calificación promedio y las estrategias de aprendizaje no variaron estadísticamente entre géneros, resultado que difiere de las investigaciones de Avendaño et al. (2016) y Acevedo et al. (2015). 
Es conveniente que los estudiantes desarrollen un profundo diálogo interior, lleven a la práctica y se retroalimenten del conocimiento previo, y a su vez lo correlacionen con los nuevos que se adquieren para que el aprendizaje se produzca de forma autónoma y autoregulada (Yang y Qian, 2018). Corresponde a los estudiantes estructurar y cumplir su propio plan de vida, con objetivos y metas bien delimitados, además de intencionales y propositivos. La finalidad de todo docente es favorecer de forma constructivista la asistencia a clase, la atención en clase, la autonomía responsable y el aprendizaje significativo, motivado y productivo de sus discípulos; para ello se provee de instrumentos, técnicas y estrategias para alcanzar los objetivos de aprendizaje y su evaluación. En la actualidad se promueve el trabajo colaborativo y grupal que estimula la autoestima, las relaciones interpersonales y la comunicación social.

\section{RECOMENDACIONES}

El presente estudio sugiere las siguientes estrategias a desarrollar con los estudiantes (1) fomentar el contacto estudiante-docente con el propósito de proveerlos de herramientas para que puedan elegir y desarrollar las estrategias de aprendizaje que más se adapten a sus necesidades; (2) implementar talleres didácticos para proveerlos de las técnicas de estudio individual y grupales, así como la concientización hacia el estudio sistemático en beneficio del aprendizaje y del rendimiento académico; (3) incrementar la motivación y la participación en clase a través del uso de nuevas tecnologías; y (4) fomentar el aprendizaje a través de retos o metas.

\section{CONCLUSIONES}

El estudio que se realiza con los estudiantes de primer semestre de Ingeniería Agropecuaria demuestra que: (1) el estilo de aprendizaje preferente es el reflexivo, con un equilibrio entre el número de estudiantes pragmáticos-teóricos-activos (Objetivo 1); (2) los hombres no presentan un estilo de aprendizaje preferente al existir estabilidad entre los estilos reflexivo, pragmático y teórico; mientras que las mujeres fueron pluralmente reflexivas y el resto no presenta un estilo de aprendizaje preferencial con valores análogos para la combinación pragmático-activo-teórico (Objetivo 1); (3) las estrategias de aprendizaje cooperativo, profundo y autónomo están en fase inicial de desarrollo (Objetivo 2), con independencia del género y la edad, excepto para el aprendizaje cooperativo en relación a la edad; (4) la metodología de aula invertida presenta un impacto significativo y provechoso en su rendimiento académico (Objetivo 3), sin mostrar variación significativa en relación al género y la edad; (5) el nivel de satisfacción es estable durante todo el semestre académico, sin existir un método pedagógico de preferencia para los estudiantes (Objetivo 4).

\section{AGRADECIMIENTOS}

Se agradece la colaboración en esta investigación a los estudiantes de primer semestre de la carrera de Ingeniería Agropecuaria, y a los Profesores Michel Laurin, PhD. y Simon Leib, MSc. por las correcciones al resumen en inglés. Las sugerencias de los revisores anónimos contribuyeron a la mejora de la calidad del manuscrito. La presente investigación se financia con los fondos PED-BIO-2015 de la autora.

\section{REFERENCIAS}

Acevedo, D., M. Durán y A. Alvis, Identificación de Estrategias de Aprendizaje de Estudiantes de Ingeniería de Alimentos en los Cursos Balance de Materia, Transporte de Fluidos y Operaciones Unitarias, doi: 10.4067/S071850062015000600005, Formación Universitaria , 8(6), 31-38 (2015)

Ali, A.R., M.E. Toriman y M.B. Gasim, Academic Achievement in Biology with Suggested Solutions in Selected Secondary Schools in Kano State, Nigeria, International J. of Education and Research, 2(11), 15-224 (2014)

Alonso, C., D. Gallego y P. Honey, Los Estilos de Aprendizaje, Mensajero, Bilbao, España (1999)

Arbizu, F. y C. Lobato, El Proceso Enseñanza-Aprendizaje en la Universidad del País Vasco: demandas y necesidades docentes desde la perspectiva del alumnado, Revista de Psicodidáctica, 15-16, 171-188 (2003)

Arroyo, A., S.P. Gil, A.L. Pascualides y L. Seisdedos, Alumnos recursantes de Botánica Agrícola I. Un análisis descriptivo de esta situación, Revista de la Facultad de Agronomía, 29(3), 191-196 (2009)

Atxurra, C., L. Villardón-Gallego y E. Calvete, Diseño y validación de la Escala de Aplicación del Aprendizaje Cooperativo (CLAS), doi: 10.1387/RevPsicodidact.11917, Revista de Psicodidáctica, 20(2), 339-357 (2015)

Avendaño, C.A., K.A. Gutiérrez, C.F. Salgado y M. Alonso-Dos-Santos, Rendimiento Académico en Estudiantes de Ingeniería Comercial: Modelo por Competencias y Factores de Influencia, doi: 10.4067/S0718-50062016000300002, Formación Universitaria, 9(3), 3-10 (2016) 
Bermúdez, G.M., S. Díaz y A.L. De Longhi, Native plant naming by high-school students of different socioeconomic status: implications for botany education, doi:10.1080/09500693.2017.1397297, International Journal of Science Education , 40(1), 46-66 (2018)

Bermúdez, G.M.A, M.E. Rivero y otros tres autores, Las clases de Biología II: Una mirada sobre la transposición, el currículum, la comunicación y la evaluación, Revista Facultad de Ciencias Exactas, Físicas y Naturales, 4(1), 89-100 (2017)

Black, P. y D. Wiliam, Classroom assessment and pedagogy, doi.org/10.1080/0969594X.2018.1441807, Assessment in Education: Principles, Policy \& Practice, 25(6), 551-575 (2018)

Buchholtz, N.F., N. Krosanke, A.B. Orschulik y K. Vorhölter, Combining and integrating formative and summative assessment in mathematics teacher education, doi: 10.1007/s11858-018-0948-y, ZDM, 50(4), 715-728 (2018)

Byng, J.W., E.F. Smets y otros seis autores, The phylogeny of angiosperms poster: a visual summary of APG IV family relationships and floral diversity, The Global Flora, 1, 1-35, Plant Gateway, Bradford, U.K. (2018)

Chalmers, C., E. Mowat y M. Chapman, Marking and providing feedback face-to-face: Staff and student perspectives, doi: 10.1177\%2F1469787417721363, Active Learning in Higher Education, 19, 35-45 (2017)

Dinah, C.S., Factors which influence academic performance in biology in Kenya: a perspective for global competitiveness, International Journal of Current Research, 5(12), 4296-4300 (2013)

Durán, J.E., A. May y D.C. Ramírez, Impacto de prácticas docentes y rendimiento académico en el aprendizaje invertido, Revista de Investigación Educativa de la Escuela de Graduados en Educación, 7(14), $50-55$ (2017)

Dytham, C., Choosing and using statistics. A biologist's guide, $2^{\text {nd }}$ Ed., Blackwell, Oxford, U.K. (2003)

Florence, W.M., A cross-sectional study: Collaborative learning approach enhances learning attitudes of undergraduate nursing students, doi: 10.5176/2345-718X_5.1.167, GSTF Journal of Nursing and Health Care (JNHC), 5(1), 1-5 (2017)

García-Vázquez, O. y A.R. Méndez-Pupo, Hacia una resignificación de la enseñanza del contenido del concepto de biodiversidad en Biología, Roca, 13(1), 158-170 (2017)

Gargallo, B., P.R. Garfella y E.C. Pérez, Enfoques de aprendizaje y rendimiento académico en estudiantes universitarios, Bordón, 58(3), 45-61 (2006)

Guerrero, R.M., R. Arregui y J. Pimienta, Análisis Psicométrico para Evaluación Educativa en una Universidad ecuatoriana, CienciAmérica, 6(2), 15-20 (2017)

Hacisalihoglu, G., D. Stephens, L. Johnson y M. Edington, The use of an active learning approach in a SCALE-UP learning space improves academic performance in undergraduate General Biology, doi. org/10.1371/journal.pone.0197916, PLoS ONE, 13(5), e0197916 (2018)

Jensen, J.L., E.A. Holt y otros tres autores, Investigating Strategies for Pre-Class Content Learning in a Flipped Classroom, doi.org/10.1007/s10956-018-9740-6, Journal of Science Education and Technology, 27(6), 523-535 (2018)

Lazzari, G., F. Gonzatti, J. M. Scopel y L. Scur, Trilha ecológica: um recurso pedagógico no ensino da Botânica, doi: 10.18226/23185279.v5iss3p161, Scientia Cum industria, 5(3), 161-167 (2017)

Limniou, M., I. Schermbrucker y M. Lyons, Traditional and flipped classroom approaches delivered by two different teachers: the student perspective, doi: 10.1007/s10639-017-9636-8, Educ. Inf. Technol., 23, 797-817 (2018)

López-Aguado, M., Diseño y análisis del Cuestionario de Estrategias de Trabajo Autónomo (CETA) para estudiantes universitarios, Revista de Psicodidáctica, 15(1), 77-99 (2010)

Montilla, C., El bosque La Ciénaga como aula ambiental para el aprendizaje de la biodiversidad en las Ciencias Naturales, Scientia Unellezea, 3(2), 49-65 (2008)

Morales-Maure, L., O. García-Marimón, A. Torres-Rodríguez y A. Lebrija-Trejos, Habilidades Cognitivas a través de la Estrategia de Aprendizaje Cooperativo y Perfeccionamiento Epistemológico en Matemática de Estudiantes de Primer Año de Universidad, doi: 10.4067/S0718-50062018000200045, Formación Universitaria, 11(2), 45-56 (2018)

Müller, N.M. у T. Seufert, Effects of self-regulation prompts in hypermedia learning on learning performance and selfefficacy, doi: 10.1016/j.learninstruc.2018.04.011, Learning and Instruction, 58, 1-11 (2018)

Pacheco, R. y E. Maldonado, Relación entre los estilos de aprendizaje y las teorías de enseñanza, Retos, 32, 7-13 (2017)

Pérez, C., R. Luengas y R.Y. Amador, Importancia de las prácticas de laboratorio en la enseñanza de la Biotecnología, Revista Teckne, 4, 26-29 (2008)

Rivero-Guerra, A.O., Evaluation of the Linnaeus' concept of Santolina rosmarinifolia L. (Asteraceae: Anthemideae) and its interpretation, Adansonia, 35, 87-105, (2013)

Rivero-Guerra, A.O., Práctica de Laboratorio de Granos de Almidón (Botánica General): una Experiencia de Clase Invertida, doi: 10.4067/S0718-50062018000100087, Formación Universitaria, 11(1), 87-104 (2018) 
Rodríguez, H.J.P., J. Robles y otros seis autores, Análisis de los estilos de aprendizaje en alumnos de Medicina de la Universidad de Chile, doi: 10.1016/j.edumed.2016.11.004, Educación Médica, 121, 1-7 (2017)

Sabater-Mateu, M.P., J.J. Curto-García y otros cinco autores, Aula invertida: experiencia en el Grado de Enfermería, Revista d'Innovació Docent Universitària, 9, 115-123 (2017)

Santos da Silva, J.R., F. Guimarães y P.T. Sano, Teaching of Botany in higher education: representations and discussions of undergraduate students, Revista Electrónica de Enseñanza de las Ciencias, 15(3), 380-393 (2016)

Shelton-Strong, S., From Learning Styles to Learning Strategies: Fostering the Capacity for Learner Autonomy, CELE Journal, (25), 121-160 (2017)

Silva, W.F., J. Chiquillo-Rodelo y C.J. Brito-Carrillo, Extrinsic Factors and their Association with the Motivation and SelfRegulation of Learning in Engineering Students, doi: 10.12988/ces.2018.8383, Contemporary Engineering Sciences, 11(20), 951-958 (2018)

Solar, N.J.B., Attributions of Academic Performance among Third Year and Fourth Year Biology Major Students, doi:10.4172/2161-0487.1000210, Asia Pacific Journal of Multidisciplinary Research, 3(3), $31-38$ (2015)

Suazo, I.C., Estilos de aprendizaje y su correlación con el rendimiento académico en anatomía humana normal, doi:10.4067/S0717-95022007000200022, International Journal of Morphology, 25(2), 367-373 (2007)

Uriarte, J. de D., Los efectos del autoconcepto académico en los procesos de aprendizaje cooperativo, Revista de Psicodidáctica, 1, 193-202 (1996)

Williams, C., S. Perlis, J. Gaughan y S. Phadtare, Creation and Implementation of a Flipped Jigsaw Activity to Stimulate Interest in Biochemistry among Medical Students, doi: 10.1002/bmb.21126, Biochemistry and Molecular Biology Education, 46, 343-353 (2018)

Yan, L.I.U., Reforms and practices of experimental teaching in plant systematics and taxonomy, Journal of Biology, 5, $024(2014)$

Yang, Y. y Q. Jing, Cooperative Learning Practices in University Classrooms, doi:10.12783/dtssehs/esem2018/23868, DEStech Transactions on Social Science, Education and Human Science, 48(1), 103-122 (2018) 